\title{
Culture fisheries in village ponds: a multi-location study in Haryana, India
}

\section{Anita Bhatnagar and Gajender Singh}

\author{
Department of Zoology, Kurukshetra University, Kurukshetra, - 136119 India \\ E:mail : anitabhatnagar@gmail.com
}

\begin{abstract}
The state of Haryana in northeastern India has an excellent sub-tropical climate for the development of fresh water fish culture in a variety of aquatic bodies. Aquaculture not only plays an important role in nutrition but also in the rural economy of the State. The main purpose of the present study was to identify the primary production and fish production patterns in village ponds under different management practices. With a more or less narrow range of primary production, varying fish production and growth rates were recorded, indicating the influence of a combination of environmental and management factors. There was no close relation between primary production and fish production in the rural fish ponds of Haryana, India. Sudden and considerable fluctuations in dissolved oxygen concentration and $\mathrm{pH}$ impair the proper functioning of other trophic communities, supported the dominance of decomposition processes, i.e., anaerobiosis, and lead to further degradation and loss of the control functions of the whole water ecosystem. Increased organic load can be considered as a general signal of reaching the instability of aquatic ecosystem and decrease of production efficiency The optimum conditions for increased fish productivity were found to be the adequate DO level $\left(>4 \mathrm{mg} \mathrm{L}^{-1}\right)$ and appropriate $\mathrm{pH}(6<\mathrm{pH}<9)$, low BOD $\left(<1.6 \mathrm{mg} \mathrm{L}^{-1}\right)$ and ammonia $\left(<0.2 \mathrm{mg} \mathrm{L}^{-1}\right)$ and optimum plankton population (approximately 3000-4500 Nos. $\mathrm{L}^{-1}$ ).
\end{abstract}

Keywords: Ammonia, BOD, Culture Fishery, Managed ponds, Pond Ecosystem, Water Quality

\section{INTRODUCTION}

Fish production in India has been growing steadily over the years and today stands at 4.5 million tones from 2.4 million tones in the late $70 \mathrm{~s}$ and at present India ranks third in fish production in the world. The dietary habits of people all over the world are creating a spurt in demand for fish particularly on account of the health conscious consumer. Haryana state with its immense water resources has tremendous scope for augmenting fish production. In 1966 the area involved in fish culture was 58 hectare and the fish production was 600 metric tones. Now in 2009 with the efforts of scientists and fisheries department the area involved in fish culture has reached 14304 hectare fish culture area and 76000 metric tonnes fish production. (According to the fisheries Department). During last decades, the state has utilized its closed water bodies viz ponds and tanks through scientific aquaculture. Pond water is considered as the major sources for fishery in Haryana of India. There are many artificial ponds and temporary water bodies of large and small size, which make an interesting biotype for physicochemical studies. It is well known that productivity of fish ponds depends largely on the abundance of fish food organisms and also on occurrence of congenial environmental condition in the pond. Optimum fish yield or sustainable production in ponds is attributable to pond functioning where in minerals and fertilizers are converted into primary producers and these serving as food base for fish growth in semi-intensive carp culture. Human interference is becoming more and more in present day aquaculture system with increasing management practices. No doubt, the system provides opportunity for increasing fish production even in small tanks but is also causing alarm to the fish culturists due to the deteriorating fragile pond ecosystem. The details of pond ecosystem have been studied by a host of workers (Delince, 1992; Garg \& Bhatnagar, 1999, 2000; Bhatnagar \& Garg, 2000; Bhatnagar, 2008 and Bhatnagar and Singh, 2010a, b). They observed that with the intensification of pond culture, perfect accounts of physico-chemical and biological aspects are not available and no such type of studies on pond fish culture in relation to water quality have been carried out in Haryana. Therefore, the present studies have been conducted, focusing monitoring of water quality and fish food organism in relation to fish growth / productivity from fish culture ponds. 


\section{MATERIALS AND METHODS}

Study Area: A general survey of the fish culture ponds in different districts of Haryana, India was made and four districts viz. Yamunanagar, Kurukshetra, Hisar and Nuh Mewat were selected on the basis of productivity. A total of fourteen fish culture ponds were selected, seven are wild (where there is no restriction on cattle entry and sewage from non-point sources also enters in the pond) and seven are managed and constructed ponds where cattle visit is limited and liming is a regular feature. Both types of ponds receive direct sunlight, the bottom soil of which contains clay.

Fish Growth studies: Fish were bulk weighed by repeated netting on bimonthly basis and specific growth rate and growth per cent gain in body weight was calculated according to following formula. Fish yield was recorded at the time of harvesting by the actual harvests.

Specific growth rate $=\ln \mathrm{W}_{2}-\ln \mathrm{W}_{1} / \mathrm{t} \times 100$

Growth per cent gain in Body weight $=W_{2}-W_{1} / W_{1}$

Where $W_{1}=$ Initial weight $W_{2}=$ Final weight $t=$ Days of experiment.

Water Quality Monitoring: Water samples were collected using plastic bottle of capacity two litres at bimonthly interval. The physico-chemical characteristics viz. temperature, dissolved oxygen, free $\mathrm{CO}_{2}$ and alkalinity were analysed at the site itself, while parameters such as hardness, calcium, magnesium, chloride, orthophosphate and total phosphate were analysed in the laboratory in accordance with NEERI (1986) and APHA (1998) on the following 2-3 days during which samples were kept in Cold. Multiline F-set 3 (E- Merck,Germany) was used for the determination of $\mathrm{DO}, \mathrm{pH}$, conductivity and salinity. BOD was estimated by seeding method. Water samples were diluted by adding distilled water and incubated for five days in BOD incubator (APHA, 1998).

Determination of Plankton density: Plankton samples were also collected by passing $25 \mathrm{~L}$ of water taken from five different locations $(5 \mathrm{~L}$ from each location) of each pond through plankton net (mesh size $50 \mu \mathrm{m}$ ) at an interval of 60 days. The samples were then carefully transferred to a measuring cylinder and a volume of $50 \mathrm{ml}$ with distilled water was made and preserved in small plastic bottles with 5 per cent buffered formalin (concentrated sample). Plankton numbers were estimated using Sedgwick Rafter cell. Identification of plankton to genus level was carried out using the keys of Ward and Whipple (1959), Prescott (1962) and Bellinger (1992). Plankton species diversity (d) was determined using the diversity index formula of Shannon and Weaver (Washington 1984).

$$
\mathrm{d}=-\Sigma(\mathrm{ni} / \mathrm{N}) \log _{2}(\mathrm{ni} / \mathrm{N})
$$

Where, $d=$ species diversity

$\mathrm{n}_{\mathrm{i}}=$ no. of individuals of $\mathrm{i}^{\text {th }}$ species

$\mathrm{N}=$ total no. of individuals

Sediment Chemistry: Sediment samples were collected from the pond bottom using cone sampler and analyzed according to Garg et al. (2002)

Statistical analysis:The coefficient of correlation " $r$ " between different water quality parameters was calculated using SPSS packages while group means were compared by students't' test (Snedecor and Cochran, 1980).

\section{RESULTS AND DISCUSSION}

Table 1 depicts the characteristics of the ponds selected. Water quality characteristics of the pond water are depicted in Table 2(Wild ponds) and Table 3 (Managed ponds). Water temperature followed the trend of atmospheric temperature being low during winter and high during summer $\mathrm{pH}$ was alkaline throughout the study period and was high in wild ponds in comparison to managed ponds. Conductivity, alkalinity, hardness, calcium, chlorides, BOD, phosphates, ammonia and plankton population all were significantly high whereas DO was low in wilds ponds in comparison to managed ponds.

NPP was significantly $(P<0.05)$ high in wild ponds. Fish yield and Specific Growth rate (Fig. 1) showed higher values in managed pond. Total fish production per year varied between 2600and $5625 \mathrm{Kg}$ per hectare in wild ponds and 5625 and $8235 \mathrm{~kg}$ per hectare in managed ponds. NPP and fish yield showed a significant negative correlation with BOD and ammonia showing that $\mathrm{BOD}$ and $\mathrm{NH}_{4}-\mathrm{N}$ are the two factors affecting the pond productivity. Biological analysis of data further revealed that total plankton population was high in wild ponds, whereas species diversity was high in managed ponds in comparison to wild ponds. Sediment chemistry of the selected ponds (Table 4) also revealed that the $\mathrm{pH}$ is throughout alkaline. The accumulation of organic carbon and conductivity is high in wild ponds in comparison to managed ponds. Nitrate $\mathrm{mg} \mathrm{Kg}^{-1}$ dry weight also revealed a similar trend. 
The studies showed that most of the ponds in Haryana are dug up common village ponds and canal fed or the farmers have tube-well as source of water. The consumption of fish in the region is increasing, and also there is an increasing demand for fresh fish from nearby cities and Delhi. This demand has led to sudden rise in the number of persons bidding for these ponds in the area, which is seen in the dramatic rise in the lease amounts. The panchayats take care of these ponds and are responsible for leasing out the ponds through open bidding process

Table 1 Selected stations and their characteristics

\begin{tabular}{|c|c|c|c|c|c|c|}
\hline Pond No. & $\begin{array}{l}\text { Pond site } \\
\text { (Name of village } \\
\text { and district) }\end{array}$ & Type & $\begin{array}{l}\text { Area } \\
\text { hectare }\end{array}$ & $\begin{array}{l}\text { Source of } \\
\text { water }\end{array}$ & Species cultured & Fertiliser used \\
\hline (1) & $\begin{array}{l}\text { Barwa } \\
\text { Kurukshetra }\end{array}$ & Wild & 0.48 & Canal & Four $(\mathrm{C}, \mathrm{R}, \mathrm{M}, \mathrm{CC})$ & No \\
\hline (2) & $\begin{array}{l}\text { Durala } \\
\text { Kurukshetra }\end{array}$ & Wild & 3.2 & $\begin{array}{l}\text { Canal / } \\
\text { Natural }\end{array}$ & Five(C,R,M,GC,CC) & No \\
\hline (3) & $\begin{array}{l}\text { Mugalpura } \\
\text { Hisar }\end{array}$ & Wild & 2.0 & $\begin{array}{l}\text { Natural/ } \\
\text { tubewell }\end{array}$ & Three $(\mathrm{C}, \mathrm{R}, \mathrm{M})$ & No \\
\hline (4) & $\begin{array}{l}\text { Jajanwala } \\
\text { Hisar }\end{array}$ & Wild & 2.0 & Canal /Natural & Four $(\mathrm{C}, \mathrm{R}, \mathrm{M}, \mathrm{CC})$ & No \\
\hline (5) & $\begin{array}{l}\text { Ghasara Tali } \\
\text { Nuh Mewat. }\end{array}$ & Wild & 3.2 & $\begin{array}{l}\text { Canal / } \\
\text { Natural }\end{array}$ & Five(C,R,M, CC,GC) & No \\
\hline (6) & $\begin{array}{l}\text { Sasoli } \\
\text { Yamunanagar. }\end{array}$ & Wild & 1.32 & $\begin{array}{l}\text { Tubewell } \\
\text { /Natural }\end{array}$ & $\operatorname{Six}(C, R, M, C C, S C, G C)$ & No \\
\hline (7) & $\begin{array}{l}\text { Chaneti } \\
\text { Yamunanagar }\end{array}$ & Wild & 1.0 & $\begin{array}{l}\text { Tubewell / } \\
\text { Natural }\end{array}$ & $\operatorname{Six}(C, R, M, C C, S C, G C)$ & No \\
\hline (1) & $\begin{array}{l}\text { Adhon } \\
\text { Kurukshetra }\end{array}$ & Cultivated & 0.6 & $\begin{array}{l}\text { Tube well / } \\
\text { canal }\end{array}$ & Four $(\mathrm{C}, \mathrm{R}, \mathrm{M}, \mathrm{CC})$ & $\begin{array}{l}\text { Cow-dung } \\
\text { Urea SSP } \\
\text { MOC }\end{array}$ \\
\hline$(2)$ & $\begin{array}{l}\text { Bohar sadan } \\
\text { Kurukshetra }\end{array}$ & Cultivated & 0.6 & $\begin{array}{l}\text { Tubewell / } \\
\text { Canal }\end{array}$ & Four $(\mathrm{C}, \mathrm{R}, \mathrm{M}, \mathrm{CC})$ & $\begin{array}{l}\text { Cow-dung } \\
\text { Urea, MOC }\end{array}$ \\
\hline (3) & $\begin{array}{l}\text { Dabra } \\
\text { Hisar }\end{array}$ & Cultivated & 0.6 & Tubewell & Three $(\mathrm{R}, \mathrm{M}, \mathrm{CC})$ & $\begin{array}{l}\text { Cow-dung, } \\
\text { urea, } \\
\text { SSP,MOC }\end{array}$ \\
\hline$(4)$ & $\begin{array}{l}\text { Satrod } \\
\text { Hisar }\end{array}$ & Cultivated & 1.2 & Tubewell & Four $(\mathrm{C}, \mathrm{R}, \mathrm{M}, \mathrm{CC})$ & $\begin{array}{l}\text { Cow-dung, } \\
\text { MOC, Rice- } \\
\text { bran }\end{array}$ \\
\hline (5) & $\begin{array}{l}\text { Ghasara } \\
\text { Nuh Mewat }\end{array}$ & cultivated & 1.2 & $\begin{array}{l}\text { Canal / } \\
\text { Tubewell }\end{array}$ & Five $(C, R, M, C C, G C)$ & $\begin{array}{l}\text { Cow-dung, } \\
\text { urea } \\
\text { SSP,MOC, }\end{array}$ \\
\hline (6) & $\begin{array}{l}\text { Hirmuthala } \\
\text { Nuh Mewat }\end{array}$ & Cultivated & 1.6 & $\begin{array}{l}\text { Canal / } \\
\text { Tubewell }\end{array}$ & Four $(\mathrm{C}, \mathrm{R}, \mathrm{M}, \mathrm{CC})$ & $\begin{array}{l}\text { Cow-dung, } \\
\text { Urea, } \\
\text { SSP,MOC, } \\
\text { Rice-bran }\end{array}$ \\
\hline (7) & $\begin{array}{l}\text { Fatehgarh } \\
\text { Yamunanagar }\end{array}$ & cultivated & $\begin{array}{l}0.44 \\
0.24\end{array}$ & Tubewell & $\operatorname{Six}(C, R, M, C C, S C, G C)$ & $\begin{array}{l}\text { Cow-dung } \\
\text {,urea, SSP } \\
\text { MOC, Rice- } \\
\text { bran }\end{array}$ \\
\hline
\end{tabular}


Agric. Biol. J. N. Am., 2010, 1(5): 961-968

Table 2. Water quality characteristics of Wild ponds ( \pm S.E. of Mean)

\begin{tabular}{|c|c|c|c|c|c|c|c|}
\hline Parameter & 1 & 2 & 3 & 4 & 5 & 6 & 7 \\
\hline Water Temp. ${ }^{\circ} \mathrm{C}$ & $22.09 \pm 1.09$ & $22.46 \pm 1.24$ & $22.12 \pm 1.40$ & $22.24 \pm 1.36$ & $22.9 \pm .86$ & $22.64 \pm 1.81$ & $22.84 \pm 1.75$ \\
\hline $\mathrm{pH}$ & $9.22 \pm 0.07$ & $8.91 \pm 0.17$ & $9.08 \pm 0.09$ & $8.19 \pm 0.02$ & $9.2 \pm 0.03$ & $8.65 \pm 0.20$ & $8.40 \pm 0.23$ \\
\hline $\begin{array}{l}\text { Conductivity } \\
\mu \mathrm{S} \mathrm{cm}^{-1}\end{array}$ & $838.88 \pm 9.01$ & $1016.49 \pm 9.12$ & $4201.94 \pm 56.60$ & $1618.33 \pm 10.45$ & $5289.6 \pm 71.01$ & $943.22 \pm 31.32$ & $681.62 \pm 37.95$ \\
\hline $\begin{array}{l}\text { Dissolved } \\
\text { oxygen } \mathrm{mg} \mathrm{L}^{-1}\end{array}$ & $5.92 \pm 0.37$ & $5.39 \pm 0.20$ & $3.93 \pm 0.08$ & $6.26 \pm 0.03$ & $7.94 \pm 0.64$ & $4.35 \pm 0.49$ & $7.30 \pm 0.89$ \\
\hline $\begin{array}{l}\text { Free } \mathrm{CO}_{2} \\
\mathrm{mg} \mathrm{L}^{-1}\end{array}$ & $12.49 \pm 2.43$ & $1.38 \pm 1.27$ & Absent & $22.55 \pm 1.42$ & Absent & $23.96 \pm 1.48$ & $28.10 \pm 3.99$ \\
\hline $\begin{array}{l}\text { Carbonate } \\
\text { Alkalinity }\end{array}$ & $3.44 \pm 3.15$ & $17.61 \pm 1.90$ & $57.21 \pm 4.25$ & 0.0 & $25.53 \pm 1.39$ & $14.00 \pm 3.68$ & 0.0 \\
\hline $\begin{array}{l}\text { Biocarbonate } \\
\text { Alkalinity }\end{array}$ & $274.94 \pm 18.67$ & $461.05 \pm 14.68$ & $429.77 \pm 25.64$ & $382.83 \pm 14.67$ & $327.46 \pm 11.33$ & $324.00 \pm 26.19$ & $264.33 \pm 12.42$ \\
\hline $\begin{array}{l}\text { Total alkalinity } \\
\mathrm{mg} \mathrm{L}^{-1}\end{array}$ & $278.38 \pm 19.05$ & $478.66 \pm 20.09$ & $486.98 \pm 22.82$ & $382.83 \pm 14.67$ & $352.99 \pm 13.44$ & $338.26 \pm 24.29$ & $264.33 \pm 12.42$ \\
\hline $\begin{array}{l}\text { Total Hardness } \\
\mathrm{mg} \mathrm{L}^{-1}\end{array}$ & $237.35 \pm 4.60$ & $276.27 \pm 17.72$ & $543.72 \pm 14.60$ & $206.33 \pm 6.60$ & $466.13 \pm 14.59$ & $218.66 \pm 13.76$ & $185.83 \pm 2.67$ \\
\hline Calcium $\mathrm{mg} \mathrm{L}^{-1}$ & $44.61 \pm 2.87$ & $40.62 \pm 4.96$ & $73.26 \pm 10.65$ & $41.57 \pm 1.69$ & $69.80 \pm 1.18$ & $19.18 \pm 1.29$ & $23.18 \pm 1.94$ \\
\hline $\begin{array}{l}\text { Magnesium } \\
\mathrm{mg} \mathrm{L}^{-1}\end{array}$ & $30.68 \pm 1.58$ & $42.65 \pm 1.51$ & $87.96 \pm 8.88$ & $25.04 \pm 2.40$ & $71.15 \pm 3.38$ & $42.43 \pm 3.50$ & $33.48 \pm 1.49$ \\
\hline $\begin{array}{l}\text { Chloride } \\
\mathrm{mg} \mathrm{L}^{-1}\end{array}$ & $64.68 \pm 6.78$ & $89.31 \pm 1.94$ & $221.26 \pm 11.16$ & $193.18 \pm 13.04$ & $103.75 \pm 1.80$ & $71.15 \pm 17.03$ & $59.3 \pm 2.72$ \\
\hline $\begin{array}{l}\text { o-phosphate } \\
\mathrm{mg} \mathrm{L}^{-1}\end{array}$ & $1.66 \pm 0.28$ & $1.41 \pm 0.41$ & $2.30 \pm 0.06$ & $1.93 \pm 0.04$ & $1.71 \pm 0.12$ & $.874 \pm 0.16$ & $1.08 \pm 0.09$ \\
\hline $\begin{array}{l}\text { Total phosphate } \\
\mathrm{mg} \mathrm{L}^{-1}\end{array}$ & $2.52 \pm 0.25$ & $2.80 \pm 0.44$ & $2.87 \pm 0.10$ & $2.54 \pm 0.03$ & $3.51 \pm 0.19$ & $1.39 \pm 0.25$ & $2.04 \pm 0.31$ \\
\hline $\begin{array}{l}\text { Total ammonia } \\
\mathrm{mg} \mathrm{L}^{-1}\end{array}$ & $1.88 \pm 0.28$ & $1.51 \pm 0.03$ & $2.05 \pm 0.56$ & $1.36 \pm 0.35$ & $3.53 \pm 0.67$ & $1.81 \pm 0.62$ & $1.285 \pm 0.07$ \\
\hline $\mathrm{BOD} \mathrm{mg} \mathrm{L}^{-1}$ & $3.04 \pm 0.08$ & $2.87 \pm 0.12$ & $2.70 \pm 0.13$ & $3.04 \pm 0.10$ & $3.01 \pm 0.08$ & $3.0 \pm 0.13$ & $2.58 \pm 0.06$ \\
\hline $\begin{array}{l}\text { Plankton } \\
\text { Population } \mathrm{L}^{-1}\end{array}$ & $\begin{array}{c}4596.66 \pm 253 . \\
71\end{array}$ & $4470.00 \pm 158.78$ & $\begin{array}{c}3780.00 \pm 193.2 \\
0\end{array}$ & $3893.33 \pm 238.60$ & $4456 \pm 176.33$ & $5030 \pm 122.24$ & $4543.33 \pm 341.66$ \\
\hline $\begin{array}{l}\text { Phytoplankton } \\
\mathrm{L}^{-1}\end{array}$ & $\begin{array}{c}2506.66 \pm 156 . \\
57\end{array}$ & $2460.00 \pm 90.65$ & $\begin{array}{c}2153.33 \pm 143.3 \\
0\end{array}$ & $2076.66 \pm 172.70$ & $2456 \pm 56.26$ & $2680 \pm 68.90$ & $2460 \pm 183.95$ \\
\hline Zooplankton $\mathrm{L}^{-1}$ & $2090 \pm 138.76$ & $2010 \pm 96.02$ & $1626.66 \pm 83.94$ & $1816.66 \pm 151.10$ & $2000 \pm 135.66$ & $2350 \pm 120.44$ & $2083.33 \pm 197.54$ \\
\hline
\end{tabular}

$\mathrm{C}=$ Catla catla; $\mathrm{R}=$ rohu (labeo rohita); $\mathrm{M}=$ mrigal (Cirrhinus mrigala)

$\mathrm{SC}=$ Silver carp; $\mathrm{GC}=$ Grass carp and $\mathrm{CC}=$ common carp 
Agric. Biol. J. N. Am., 2010, 1(5): 961-968

Table 3 Water quality characteristics of Managed Ponds ( \pm S.E. of Mean)

\begin{tabular}{|c|c|c|c|c|c|c|c|}
\hline Parameter & 1 & 2 & 3 & 4 & 5 & 6 & 7 \\
\hline $\begin{array}{l}\text { Water Temp. } \\
{ }^{0} \mathrm{C}\end{array}$ & $22.43 \pm 1.20$ & $21.07 \pm 1.60$ & $23.57 \pm 1.06$ & $23.92 \pm .90$ & $22.45 \pm 1.28$ & $22.93 \pm 1.02$ & $23.03 \pm 1.85$ \\
\hline $\mathrm{pH}$ & $8.51 \pm 0.15$ & $8.52 \pm 0.10$ & $8.17 \pm 0.08$ & $8.12 \pm 0.13$ & $8.17 \pm 0.09$ & $8.83 \pm 0.14$ & $7.65 \pm 0.13$ \\
\hline $\begin{array}{l}\text { Conductivity } \\
\mu \mathrm{S} \mathrm{cm}^{-1}\end{array}$ & $487.77 \pm 57.27$ & $625.55 \pm 47.43$ & $1023.44 \pm 84.40$ & $427.66 \pm 15.97$ & $2688.66 \pm 36.28$ & $\begin{array}{l}3576.59 \pm 39.6 \\
1\end{array}$ & $592.38 \pm 46.47$ \\
\hline $\begin{array}{l}\text { Dissolved } \\
\text { oxygen } \mathrm{mg} \mathrm{L}^{-}\end{array}$ & $7.58 \pm 0.09$ & $7.68 \pm 0.19$ & $7.21 \pm 0.05$ & $7.51 \pm 0.06$ & $8.36 \pm 0.64$ & $8.11 \pm 0.48$ & $9.38 \pm 0.90$ \\
\hline $\begin{array}{l}\text { Free } \mathrm{CO}_{2} \\
\mathrm{mg} \mathrm{L}^{-1}\end{array}$ & Absent & $14.27 \pm 3.12$ & $11.50 \pm 0.95$ & $13.10 \pm 1.55$ & Absent & Absent & $26.33 \pm 3.36$ \\
\hline $\begin{array}{l}\text { Carbonate } \\
\text { Alkalinity }\end{array}$ & $23.05 \pm 0.85$ & 0.00 & 0.00 & 0.00 & $14.06 \pm 1.74$ & $13.86 \pm 0.37$ & $13.44 \pm 3.60$ \\
\hline $\begin{array}{l}\text { Biocarbonate } \\
\text { Alkalinity }\end{array}$ & $295.05 \pm 5.89$ & $268.99 \pm 12.74$ & $240.55 \pm 9.16$ & $168.16 \pm 2.34$ & $173.33 \pm 5.51$ & $378.46 \pm 28.29$ & $265.44 \pm 12.32$ \\
\hline $\begin{array}{l}\text { Total } \\
\text { alkalinity } \\
\mathrm{mg} \mathrm{L}^{-1}\end{array}$ & $318.10 \pm 6.12$ & $268.99 \pm 12.74$ & $240.55 \pm 9.16$ & $168.16 \pm 2.34$ & $187.39 \pm 4.14$ & $392.32 \pm 28.09$ & $278.88 \pm 12.94$ \\
\hline $\begin{array}{l}\text { Total } \\
\text { Hardness } \\
\mathrm{mg} \mathrm{L}^{-1} \\
\end{array}$ & $198.71 \pm 5.39$ & $217.89 \pm 3.09$ & $234.83 \pm 28.21$ & $172.11 \pm 3.24$ & $312.73 \pm 10.99$ & $349.43 \pm 8.65$ & $231.27 \pm 16.37$ \\
\hline $\begin{array}{l}\text { Calcium } \mathrm{mg} \\
\mathrm{L}^{-1}\end{array}$ & $17.72 \pm 1.19$ & $41.49 \pm 1.60$ & $43.54 \pm 11.76$ & $26.45 \pm 4.06$ & $76.93 \pm 43.05$ & $61.65 \pm 1.94$ & $52.38 \pm 8.77$ \\
\hline $\begin{array}{l}\text { Magnesium } \\
\mathrm{mg} \mathrm{L}^{-1}\end{array}$ & $37.51 \pm 1.88$ & $27.82 \pm 1.41$ & $30.75 \pm 1.05$ & $25.87 \pm 2.79$ & $58.65 \pm 2.40$ & $47.65 \pm 1.51$ & $26.90 \pm 2.45$ \\
\hline $\begin{array}{l}\text { Chloride } \\
\mathrm{mg} \mathrm{L}^{-1}\end{array}$ & $24.59 \pm 1.72$ & $33.15 \pm 7.45$ & $115.65 \pm 22.55$ & $9.42 \pm .38$ & $90.57 \pm 2.01$ & $82.82 \pm 1.38$ & $38.04 \pm 14.78$ \\
\hline $\begin{array}{l}\text { o-phosphate } \\
\mathrm{mg} \mathrm{L}^{-1}\end{array}$ & $0.69 \pm 0.14$ & $0.60 \pm 0.06$ & $0.74 \pm 0.02$ & $0.46 \pm 0.02$ & $.962 \pm 0.18$ & $1.49 \pm 0.05$ & $.780 \pm 0.16$ \\
\hline $\begin{array}{l}\text { Total } \\
\text { phosphate } \\
\mathrm{mg} \mathrm{L}^{-1} \\
\end{array}$ & $1.17 \pm 0.22$ & $1.01 \pm 0.11$ & $1.15 \pm 0.05$ & $0.63 \pm 0.05$ & $1.62 \pm 0.14$ & $2.69 \pm 0.24$ & $1.21 \pm 0.14$ \\
\hline $\begin{array}{l}\text { Total } \\
\text { Ammonia } \\
\mathrm{mgL}^{-1}\end{array}$ & $0.29 \pm 0.02$ & $0.39 \pm 0.05$ & $0.52 \pm 0.04$ & $0.59 \pm 0.11$ & $1.29 \pm 0.18$ & $3.44 \pm 0.64$ & $.737 \pm 0.19$ \\
\hline BOD & $2.05 \pm 0.05$ & $2.03 \pm 0.06$ & $2.45 \pm 0.05$ & $2.38 \pm 0.10$ & $2.31 \pm 0.09$ & $2.70 \pm 0.09$ & $1.66 \pm 0.09$ \\
\hline $\begin{array}{l}\text { Plankton } \\
\text { Population L }{ }^{-1}\end{array}$ & $\begin{array}{l}3906.66 \pm 229.6 \\
3\end{array}$ & $\begin{array}{l}3926.66 \pm 174.8 \\
8\end{array}$ & $3520.00 \pm 208.60$ & $3333.33 \pm 156.8$ & $3992 \pm 170.31$ & $3968 \pm 232.32$ & $\begin{array}{l}4566.66 \pm 147.6 \\
6\end{array}$ \\
\hline $\begin{array}{l}\text { Phytoplankto } \\
\mathrm{n}\end{array}$ & $\begin{array}{l}2320.00 \pm 167.7 \\
1\end{array}$ & $\begin{array}{l}2420.00 \pm 104.0 \\
0\end{array}$ & $2206.66 \pm 157.7$ & $2083.33 \pm 113.0$ & $2496 \pm 106.78$ & $2392 \pm 150.67$ & $\begin{array}{l}2926.66 \pm 117.6 \\
9\end{array}$ \\
\hline Zooplankton & $1586.66 \pm 85.00$ & $1506.66 \pm 95.43$ & $1313.33 \pm 98.55$ & $1037.33 \pm 183.00$ & $1512.0 \pm 92.44$ & $1576 \pm 95.32$ & $1640 \pm 59.10$ \\
\hline
\end{tabular}


Agric. Biol. J. N. Am., 2010, 1(5): 961-968

Table 4 Soil quality parameter of wild and managed ponds

\begin{tabular}{|c|c|c|c|c|c|c|c|}
\hline \multirow{2}{*}{\multicolumn{2}{|c|}{ Pond type }} & \multicolumn{6}{|c|}{ Parameters } \\
\hline & & \% Moisture & p H & $\begin{array}{l}\text { Conductivity } \\
\left(\mu \mathrm{S} \mathrm{cm}^{-1}\right)\end{array}$ & $\begin{array}{l}\text { Available } \\
\text { phosphate (mg } \\
\mathrm{Kg}^{-1} \text { dry Wt.) }\end{array}$ & $\begin{array}{l}\text { Nitrate } \\
\left(\mathrm{mg} \mathrm{Kg}{ }^{-1} \text { dry }\right. \\
\text { Wt.) }\end{array}$ & $\begin{array}{l}\text { Organic } \\
\text { carbon \% }\end{array}$ \\
\hline \multirow{7}{*}{$\begin{array}{l}\text { Wild } \\
\text { Ponds }\end{array}$} & 1 & $29.6 \pm 1.16$ & $8.31 \pm 0.02$ & $720.0 \pm 4.36$ & $0.20 \pm 0.07$ & $0.076 \pm 0.01$ & $0.836 \pm 0.06$ \\
\hline & 2 & $28.33 \pm 1.36$ & $8.26 \pm 0.81$ & $970.7 \pm 2.99$ & $0.18 \pm 0.06$ & $0.068 \pm 0.04$ & $0.793 \pm 0.03$ \\
\hline & 3 & $31.66 \pm 4.22$ & $8.26 \pm 0.01$ & $987.6 \pm 10.7$ & $0.11 \pm 0.003$ & $0.071 \pm 0.04$ & $0.913 \pm 0.06$ \\
\hline & 4 & $33.61 \pm 2.44$ & $7.16 \pm 0.04$ & $1319.9 \pm 38.9$ & $0.16 \pm 0.003$ & $0.053 \pm 0.02$ & $0.864 \pm 0.02$ \\
\hline & 5 & $31.8 \pm 2.0$ & $7.24 \pm 0.13$ & $897.0 \pm 15.20$ & $0.03 \pm 0.001$ & $0.028 \pm 0.02$ & $0.793 \pm 0.02$ \\
\hline & 6 & $21.7 \pm 0.8$ & $7.69 \pm 0.16$ & $940.44 \pm 17.76$ & $.0 .08 \pm 2.12$ & $0.031 \pm 0.02$ & $0.712 \pm 0.01$ \\
\hline & 7 & $27.7 \pm 1.10$ & $7.09 \pm 0.18$ & $555.0 \pm 18.76$ & $0.08 \pm 0.04$ & $0.021 \pm 0.04$ & $0.606 \pm 0.02$ \\
\hline \multirow{7}{*}{$\begin{array}{l}\text { Managed } \\
\text { Ponds }\end{array}$} & 1 & $25.33 \pm 2.11$ & $7.8 \pm 0.023$ & $491.66 \pm 1.99$ & $0.085 \pm 0.05$ & $0.066 \pm 0.06$ & $0.67 \pm 0.02$ \\
\hline & 2 & $24.66 \pm 0.09$ & $7.76 \pm 0.02$ & $642.4 \pm 2.48$ & $0.06 \pm 0.06$ & $0.035 \pm 0.05$ & $0.76 \pm 0.01$ \\
\hline & 3 & $27.2 \pm 0.98$ & $8.31 \pm 0.06$ & $992.8 \pm 64.54$ & $0.05 \pm 0.003$ & $0.021 \pm 0.04$ & $0.513 \pm 0.06$ \\
\hline & 4 & $26.6 \pm 1.19$ & $7.41 \pm 0.06$ & $563.0 \pm 21.54$ & $0.06 \pm 0.06$ & $0.026 \pm 0.02$ & $0.981 \pm 0.02$ \\
\hline & 5 & $25.34 \pm 1.19$ & $7.29 \pm 0.13$ & $2729.66 \pm 12.50$ & $0.03 \pm 0.005$ & $0.03 \pm 0.01$ & $0.691 \pm 0.01$ \\
\hline & 6 & $24.33 \pm 0.8$ & $7.90 \pm 0.11$ & $3626.66 \pm 14.08$ & $0.08 \pm 0.005$ & $0.061 \pm 0.04$ & $0.809 \pm 0.03$ \\
\hline & 7 & $23.3 \pm 1.2$ & $7.02 \pm 0.30$ & $678.0 \pm .50 .00$ & $0.088 \pm 0.06$ & $0.02 \pm 0.04$ & $0.171 \pm 0.03$ \\
\hline
\end{tabular}

The fish production efficiency varied over a wide range in both wild and managed village ponds. However, the average conversion was more efficient in the managed ponds. According to the effect of fish growth on water quality the positive effect is that they stablise the food web and maintain steady production and negative effect is that leading with increase biomass a decrease in net production factor (Chand, et al 2006). Similar results have been observed in the present studies. It was further observed that fish production is strongly influenced by the inputs. There have been a number of attempts at correlating the fish yields with limnological factors influencing the productivity of water bodies (Rawson, 1955; Ryder, 1965, Garg and Bhatnagar, 1999, 2000). The distribution of hydrogen ion concentration was found to be high in wild ponds as compared to managed ponds, where high $\mathrm{pH}$, carbonate is the dominant ion and little carbon dioxide is made available by the buffer system, thus reducing photosynthesis (Boyd, 1972). Generally the values for electrical conductivity were high during summer may be because of high evaporation rates and salt concentrations (Talling and Talling, 1965). Total alkalinity was high in wild ponds as compared to managed pond. High value of total alkalinity is indicative of the productive nature of the water body suggesting that in the present studies all the ponds were in good trophic status (Jhingran, 1992 and Singh, 1998). Chlorides, total hardness, calcium and magnesium were high in wild ponds in comparison to managed ponds may be because of unmanaged entry of cattle and domestic waste, since these parameters are usually taken as an index of pollution (Hansson, 1989 ; Bhatnagar and Sanghwan, 2009 and Bhatnagar et al., 2009) . 


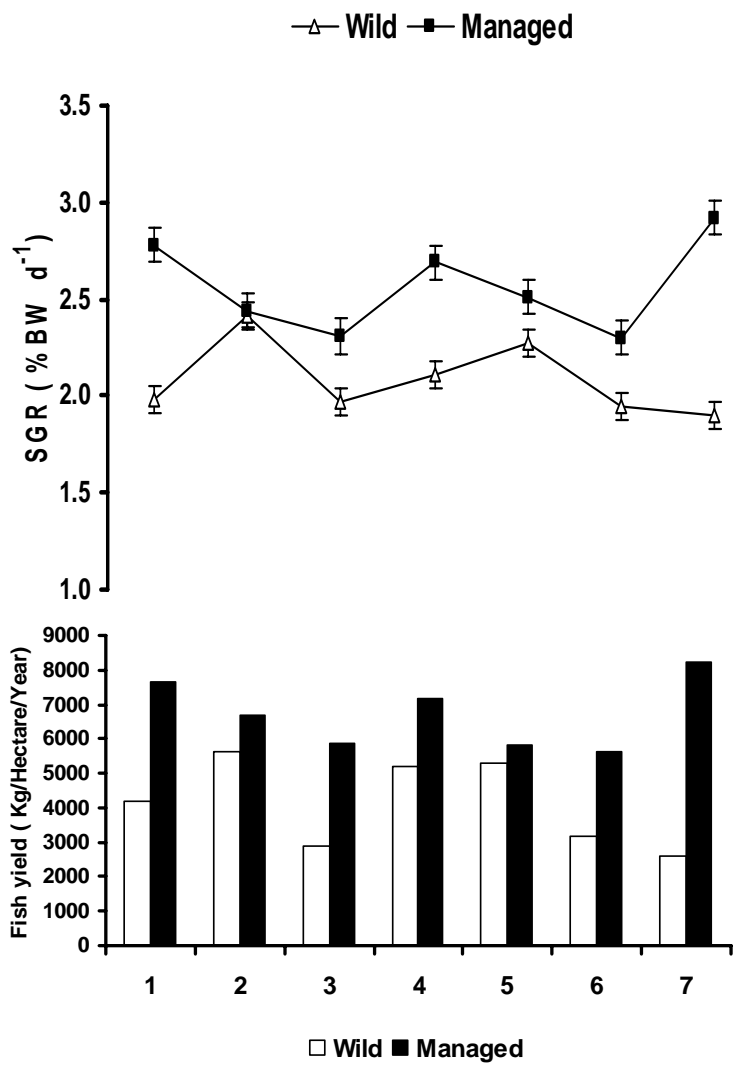

Fig. 1.Fish Yield and specific growth rate of stocked fishes in managed and wild ponds in Haryana, India

Increased chloride contents also indicate the pollution of animal origin. However, organic pollution indicating parameters BOD, Chlorides, phosphates and ammonia were high in wild ponds in comparison to managed ponds, though no fertilizers were added in wild ponds. Pond sediment also showed high level of organic pollution depicting high accumulation of organic carbon and nitrate in the pond sediment. This increase in these parameters in the wild ponds might be because of unmanaged entry of cattle and domestic waste coming from non-point sources, since these parameters are usually taken as an indicator of pollution (Bhatnagar and Sangwan, 2009; Garg and Bhatnagar, 2000, 2003, 2010b). Alkalinity and $\mathrm{pH}$ have direct effect on the orthophosphate concentration in water. A decrease in carbon-dioxide resulting from photosynthesis or gaseous diffusion, increases $\mathrm{pH}$ and cause phosphate precipitation (Hepher, 1966). Dissolved oxygen is considered, significant both as regulator of metabolic process and indicator of water quality. Low DO retaining capacity of water due to increased organism's respiratory demand at high temperature may also support these low values of dissolved oxygen (Rao, 1986). In the present study, DO remained well above the minimum level $>5 \mathrm{ppm}$ in managed ponds (Banerjea, 1967) to support good fish production. Ammonia concentration was also high in the wild ponds. According to Meade (1985), maximum limit of ammonia concentration for aquatic organisms is 0.1 $\mathrm{mg} \mathrm{L}^{-1}$ and high value of ammonia causes ammonia toxicity resulting in osmoregulatory imbalance, kidney failure and damage to gill epithelium which leads to suffocation. According to Harrison, (1978) high concentration of calcium, magnesium and phosphate ions also have a positive effect on ammonification, since ammonification is performed by heterotrophic bacteria whose density is strongly dependent on organic substrate available (Barat and Jana, 1987). No significant variations were observed in phytoplankton population in wild or managed ponds. The zooplanktons were significantly higher in wild ponds, yet the fish production was high in managed ponds. Low zooplankton population in managed ponds but high fish growth may be because of grazing pressure exerted by the fish (Delince, 1992). Since plankton production depends upon carrying capacity of environment and nutrient factors, oxygen supply in the pond and nutrient availability and supply may have direct effect on production of fish food organisms. The phytoplankton-zooplankton interface is the crucial point where changes in the predators at the top of food web are translated to changes in the property of ecosystem such as primary productivity and nutrient recycling (Elser et al, 1990). These studies clearly depicted that there still exists ample scope for further growth in this sector by managing levels of inputs and monitoring water quality Numerous constraints like seed, feed, training, markets and labour are being taken care by the state government by setting up the FFDA which provided technical assistance for growth of fishery. The lack of innovation and stagnation of the cooperative sector in fishery is a concern, mainly because it deals with the livelihoods of the marginalized community who seem to be left out from the growth of the sector.

\section{ACKNOWLEDGEMENTS:}

This study was supported by a grant from major project sanctioned by Department of Fisheries Govt. of Haryana, India. 


\section{REFERENCES}

APHA, AWWA, WPCF (1998) Standard methods for the examination of water and waste water, $20^{\text {th }}$ edn., American Public Health Association, (American Public Health Association, New York). DC20005-2605

Banerjea, S.M. (1967) Water quality and soil condition of fishponds in some states of India in relation to fish production. Indian journal of Fisheries, 14, 115-144.

Barat, S. and Jana, B.B. (1987) Effects of farming management on the distribution pattern of ammonification rate, protein mineralizing and ammonifying bacterial population in experimental culture tanks. Bamidgeh, 39: 120-132.

Bellinger, E. G. (1992) A key to common Algae. The institute of water and environmental management, London

Bhatnagar, A. and Sangwan, P. (2009) Impact of mass bathing on water quality. International Journal of Environmental Research. Iran. 3(2): 247-252.

Bhatnagar, A. and Garg, S.K. (2000) Causative factors of fish mortality in still water fish ponds under sub-tropical conditions. Aquaculture, 1(2): 91-96.

Bhatnagar, A. (2008) Productivity and fish biodiverisity of selected ponds of Haryana. Project Report submitted to Department of fisheries Government of Haryana

Bhatnagar, A, Chopra, G. and Malhotra, P. (2009) Water quality indices and abiotic characteristics of western Yamuna canal in Yamunanagar, Haryana.. Journal of Applied and Natural Science 1(2):149-154.

Bhatnagar, A. and Singh, G. (2010a). Assessment of culture fisheries in village ponds: A study in district Hisar, Haryana, India International Journal of Environmental Research. Iran. Vol.4 (1): 57-64.

Bhatnagar, A. and Singh, G. (2010b). Fish pond ecosystem: Assessment and Sustainability. National seminar on Integrated Management of water resources with reference to biodiversity and livelihood. pp72.

Boyd, C.E. (1972) Sources of $\mathrm{CO}_{2}$ for nuisance blooms of algae. Weed Science 20: 492-497.

Chand, B.K., Goswami, A., Biswas, P.K., Biswas P. and Patra B.C. (2006) Effect of stocking levels of ducks on production of Indian Major Carps in village ponds under duck-fish integrated system in West Bengal state of India. Livestock Research for Rural Development. Vol.18, Article \#6. Retrieved August 4, 2009, from http://www.Irrd.org//rrd18/1/chan18006.htm

Delince, G . (1992) The ecology of the fish pond ecosystem. Kluwer Acadmic Publisers London 230pp

Elser, J.J., Carney, H.J. and Goldman, C.R. (1990) The zooplankton-phytoplankton interface in lakes of contrasting trophic status: an experimental comparision. Hydrobiologia 200/201: 69-82.

Garg, S.K. and Bhatnagar, A. (1999) Effect of different doses of organic fertilizer (cowdung) on pond productivity and fish biomass in still water ponds. Journal of Applied Ichthylogy. (Germany)15 : 10-18

Garg, S.K. and Bhatnagar, A. (2000) Effect of fertilization frequency on pond productivity and fish biomass in still water ponds. Aquaculture Research (The Netherlands) 31: $409-414$

Garg, S.K., Bhatnagar, A., Kalla, A. and Johal, M.S. (2002) Experimental Ichthyology. CBS Publishers, New Delhi. P.149.

Garg, S.K. and Bhatnagar, A. (2003) Development of Ecofriendly Fish culture Technology. In: Proceedings of National Seminar on Emerging environmental issues and Technological challenges.Sept.01-02, 2003 GJU, Hisar.

Hansson, L.A. 1989 The influence of a periphytic biolayer on phosphorus exchange between substrate and water. Arch Hydrobiology 115: 21-26.

Harrison, W.G. (1978) Experimental measurements of nitrogen remineralization in costal waters. Limnology Oceanography 23: 684-694.

Hepher, B. (1966) Some aspects of the phosphrus cycle in fishponds. Verh. International. Ver. Limnology 16: 12931297.

Jhingran, V.G. (1992) Fish and Fisheries of India. Hidustan Publising Corporation, New Delhi, India. 666p.

Meade, J.W. (1985) Allowable ammonia for fish culture. Progressive Fish culture. 47: 135-145

NEERI. (1986) Manual on water and waste water analysis National Environmental Research Institute, Nagpur.

Prescott, G .W. (1962) Algae of the Western Great Lakes Area. Wm. C. Brown Co., Dubuque, IA.

Rao, K.J. (1986) Studies on the seasonal diurnal variations in some physico-chemical conditions of a fresh water pond under prawn culture. Proceeding of National symposium Fish and Enviornment.pp. 96-102.

Rawson, D.S. (1955) Morphometry as a dominant factor in the productivity of large lakes. International. Ver. Theor. Ange. Limnology Verh., 12:164-175.

Ryder, R.A. (1965) A method for estimating the potential fish production of north-temperate lakes. Trans. American. Fishery Society. 94:214-218

Singh, H.P. (1998) Studies on primary production in Gobindsagar reservoir, Himachal Pradesh. Journal of Environmental Biology 19, 167-170.

Snedcor, G.W. and Cochran, W.G. (1980) $7^{\text {th }}$ edn. Statistical methods. lowa State University. lowa. pp.507.

Talling, J.F. and Talling, I.B. (1965) The chemical composition of African lake waters. International Revue Ges. Hydrobiologia. 50: 421-463.

Ward , H.B. and Whipple, G.C. (1959) Freshwater Biology, John Wiley and Sons, pp. 1248.

Washington, H.G. (1984) Diversity, biotic and similarity indices. Water Research 18(6): 653-694. 